\title{
Computer Mouse Implementation using Object Detection and Image Processing
}

\author{
Ankur Yadav \\ Ideal Institute of \\ Technology, Ghaziabad, \\ India
}

\author{
Anand Pandey \\ Ideal Institute of \\ Technology, Ghaziabad, \\ India
}

\author{
Avinash Singh \\ Ideal Institute of \\ Technology, Ghaziabad, \\ India
}

\author{
Ajit Kr Singh \\ Ideal Institute of \\ Technology, Ghaziabad, \\ India
}

\begin{abstract}
There are so many devices developed to help the physical world interact with the digital world but only few are there for gesture recognition made by body movements. Gesture recognition technology is already developed. In this paper three technologies are mainly used: object detection, image processing and colour recognition for reducing the bridge between the physical world and the digital world by using Sixth sense technology. Sixth sense technology is a set of wearable devices that acts as a gestural interface between the physical world and the digital world. The system recognizes a user's free hand gestures as well as icons/symbols drawn in the air with the fingers. The aim of this paper is to move the mouse cursor on the screen without using hardware such as a mouse and only by moving the cursor through finger movements i.e. the process of gesture recognition. The implementation of the computer mouse is through the fingers by image grabbing using Sixth Sense Technology which is finally processed in MATLAB without using gesture recognition.
\end{abstract}

\section{Keywords}

Sixth Sense Technology,object Detection,colour Recognition, MATLAB, Image Processing, Mouse, Keyboard.

\section{INTRODUCTION}

Sixth Sense is a set of wearable devices that acts as a gestural interface between the digital world and the physical world. As we know that the introduction of new devices to interact with computers is not changed. This is probably because the existing devices are adequate. But now a days computers have been so tightly integrated with everyday life, that new applications along with new hardwares are constantly introduced. The means of communication with computers at the moment is limited to keyboards, mice, light pen, trackball, microphones, keypads, etc. These devices have grown to be familiar but inherently limit the speed and flexibility with which we interact with the computer. Computer recognition of hand gestures may provide a more natural-computer interface, allowing people to point, or rotate a CAD model by rotating their hands. Hand gestures can be classified in two categories: static and dynamic. A static gesture is a particular hand configuration and pose, represented by a single image. A dynamic gesture is a moving gesture, represented by a sequence of images. But we will focus on the recognition of static images [11]

In this technique, the hand movement of a user is mapped into mouse inputs. A web camera is set to take the live video continuously and then from this video various images are being captured by using the MATLAB. The user must have a particular color marker in his hand so that when the web camera takes image it must be visible in the image. This color is detected from the image pixel in MATLAB and the pixel position is mapped into mouse input by using object detection. Depending upon the size of the image taken by camera various scaling techniques are used like image analysis, image segmentation and image processing etc.

Using sixth sense technology, some other applications can also be developed, like taking the live pictures from the physical world and saving them to the system without using any hardware i.e. only by hand gestures, watching videos, drawing scenery and many more. The following figures show the examples of the applications of the same.
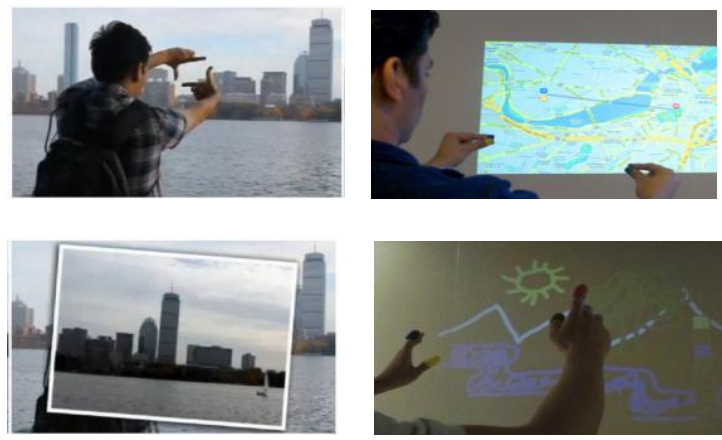

\section{METHODS \& TECHNOLOGIES}

\section{USED}

Sixth Sense is a wearable gestural interface that augments the physical world around us with digital information and lets us use natural hand gestures to interact with that information.

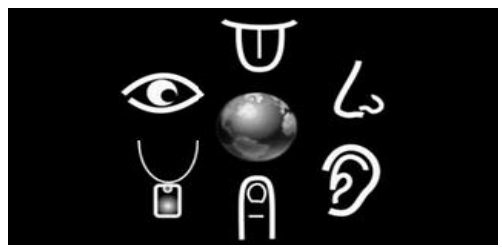

Figure: 1 Six Senses

Sixth Sense in scientific (or non-scientific) terms is defined as Extra Sensory Perception i.e. in short ESP. It involves the reception of information which is not gained through any of the five existing senses. Nor is it taken from any experiences from the past or known. Sixth Sense aims to more seamlessly integrate online information and tech into everyday life. By making available information needed for decision-making beyond what we have access to with our five senses, it effectively gives users a sixth sense[6]. 
The hardware components coupled are:

\section{Camera-}

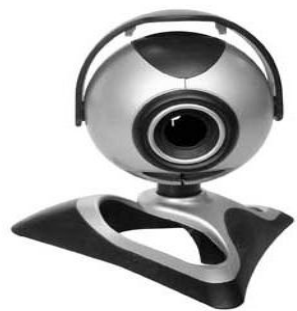

Figure: 2 Web Camera

A webcam captures and recognises an object in view and tracks the user's hand gestures using computer-vision based techniques. It sends the data to the smart phone. The camera, in a sense, acts as a digital eye, seeing what the user sees. It also tracks the movements of the thumbs and index fingers of both of the user's hands. The camera recognizes objects around you instantly, with the micro projector overlaying the information on any surface, including the object itself or your hand.

\section{Color Markers-}

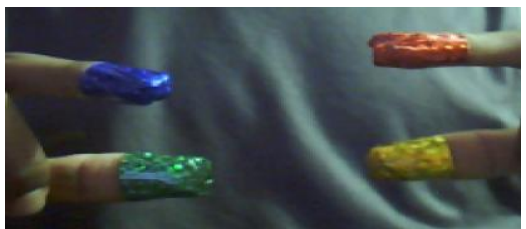

Figure: 3 Color Markers

It is at the tip of the user's fingers. Marking the user's fingers with red, yellow, green, and blue tape helps the webcam recognize gestures. The movements and arrangements of these makers are interpreted into gestures that act as interaction instructions for the projected application interfaces.

\section{Laptop/computer-}

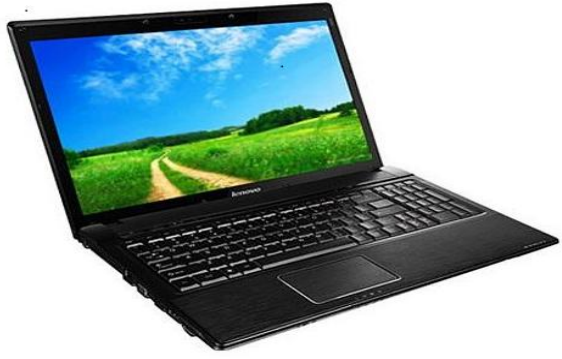

Figure: 4 Laptop

A laptop or a computer is used to send or receive data from anyone and to anyone via the internet. A Laptop runs the Sixth Sense software, and handles the connection to the internet. The software searches the Web and interprets the hand gestures.

\subsection{Gesture Recognition}

Gesture recognition is defined as interpreting human gestures using mathematical algorithms. Gestures can originate from any bodily motion or state. Current focuses in the field include emotion recognition from the face, hand gesture recognition or even body gestures. Many approaches have been made using cameras and computer vision algorithms to interpret sign language. However, the identification and recognition of human behaviours is also the subject of gesture recognition techniques. One of the important benefits of gesture recognition is that it can provide the help and assistance for people with disabilities such as the deaf or anyone who cannot use his voice to communicate or make gestures using his/her body.

It is a technology which is aimed at interpreting human gestures with the help of mathematical algorithms. Gesture recognition technique basically focuses on the emotion recognition from the face and hand gesture recognition. Gesture recognition technique enables humans to interact with computers in a more direct way without using any external interfacing devices. It can provide a much better alternative to text user interfaces and graphical user interface which requires the need of a keyboard or mouse to interact with the computer. An interface which solely depends on the gestures requires precise hand pose tracking. In the early versions of gesture recognition process special type of hand gloves which provide information about hand position orientation and flux of the fingers. In the Sixth Sense devices colored bands are used for this purpose. Once hand pose has been captured the gestures can be recognized using different techniques [6].

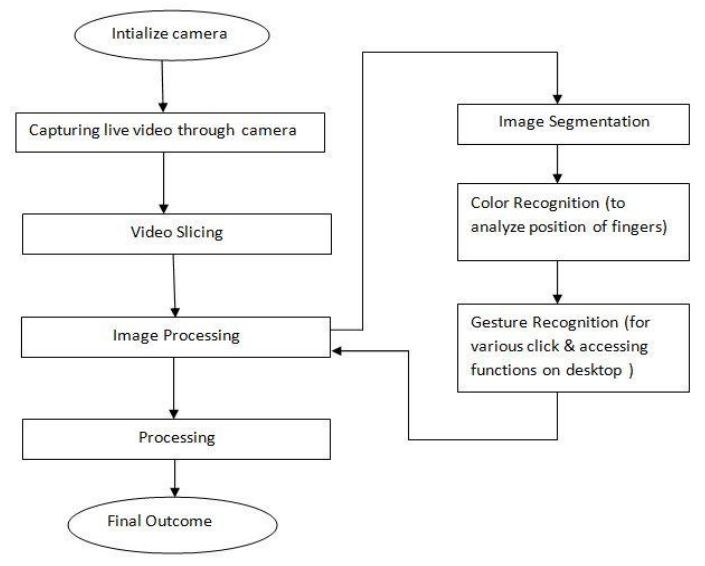

Figure: 5 System Flow chart

\section{IMAGE PROCESSING USING MATLAB}

When working with images in MATLAB, there are many things to keep in mind such so loading an image, using the right format, saving the data as different data types, how to display an image, conversion between different image formats, etc. Most of these commands require you to have the image processing tool box installed with MATLAB.

The following image formats are supposed by MATLAB:

- $\quad$ BMP

- $\mathrm{HDF}$

- JPEG

- $\quad$ PCX

- $\quad$ TIFF

- XWB

If an image is stored as a JPEG image on your disc we first read it into MATLAB. However, in order to start working with an image for example, perform a wavelet transform on the image, we must convert it into a different format. This section explains four common formats.

- Intensity image (gray scale image)

- Binary image 
- Indexed image

- $\quad$ RGB image

- Multiframe image

We mainly use the RGB format, which is a format for colour images. It represents an image with three matrices of sizes matching the image format. Each matrix corresponds to one of the colours red, green or blue and gives an instruction of how much of these colours a certain pixels should use [11].

How to convert between different formats:

The following table shows how to convert between the different formats given above. All these commands require the Image processing tool box.

Image format conversion (Within the parenthesis you type the name of the image you wish to convert.)

Table: 1 Image format conversion

\begin{tabular}{|l|l|}
\hline Operation: & $\begin{array}{l}\text { Matlab } \\
\text { command: }\end{array}$ \\
\hline $\begin{array}{l}\text { Convert bet intensity/indexed/RGB formats } \\
\text { to binary format. }\end{array}$ & dither() \\
\hline $\begin{array}{l}\text { Convert between intensity formats to } \\
\text { indexed format. }\end{array}$ & gray2ind() \\
\hline $\begin{array}{l}\text { Convert between indexed formats to } \\
\text { intensity format. }\end{array}$ & ind2gray() \\
\hline $\begin{array}{l}\text { Convert between indexed formats to RGB } \\
\text { format. }\end{array}$ & ind2rgb() \\
\hline $\begin{array}{l}\text { Convert a regular matrix to intensity format } \\
\text { by scaling. }\end{array}$ & mat2gray() \\
\hline $\begin{array}{l}\text { Convert between RGB formats to intensity } \\
\text { format. }\end{array}$ & rgb2gray() \\
\hline $\begin{array}{l}\text { Convert between RGB formats to indexed } \\
\text { format. }\end{array}$ & rgb2ind() \\
\hline
\end{tabular}

Image processing usually refers to digital image processing, but optical and analog image processing also are possible. This article is about general techniques that apply to all of them. The acquisition of images (producing the input image in the first place) is referred to as imaging.

Aspects of Image Processing:

- Image Enhancement: Processing an image so that the result is more suitable for a particular application. (Sharpening or deblurring an out of focus image, highlighting edges, improving image contrast, or brightening an image, removing noise)

- Image Restoration: This may be considered as reversing the damage done to an image by a known cause. (Removing of blur caused by linear motion, removal of optical distortions)

- Image Segmentation: This involves subdividing an image into constituent parts, or isolating certain aspects of an image. (Finding lines, circles, or particular shapes in an image, in an aerial photograph, identifying cars, trees, buildings, or roads [10].

\section{STUDY OF EXISTING SOFTWARES \& METHODS}

The method we use is based on Sixth sense technology where user has some devices such as a laptop embedded with webcam for capturing the live video of the user, a MATLAB software for processing the input and recognizing the colours at the finger tips of the user. The following figure shows the hardware devices we have used for experimental purpose. The process begins by taking a live video with the help of a camera embedded in the laptop, also
MATLAB installed in the laptop processes the input and recognizes the colours present on the finger tips of the user for the different clicks wherein each click is associated with a different colour. The figure shown below shows the approach to move mouse cursor on screen through finger movements using sixth sense technology.

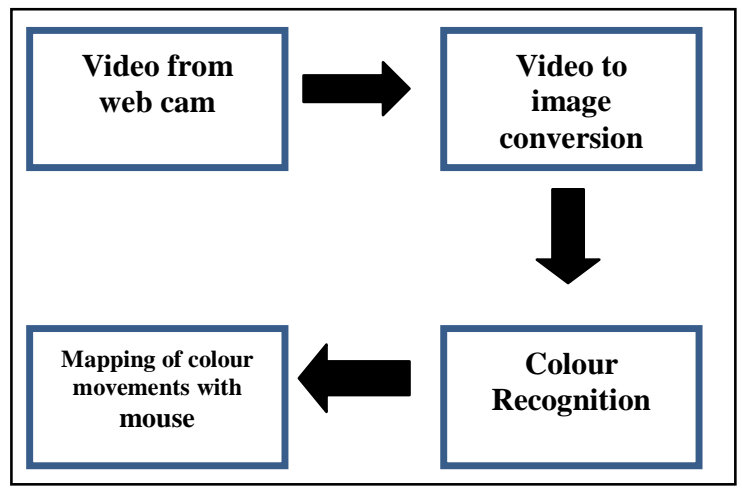

Figure: 6 Proposed Methodology

Firstly, a camera is used to interact with the physical world. Camera starts recording the live video as input, processes the input and sends the input to MATLAB installed in the laptop. In MATLAB a code is prepared which convert the incoming live video into frames of images which is also known as slicing of video. The images obtained from slicing of video is then processed for colour recognition process. The output of colour recognition process contains only those images of which colours are coded in MATLAB, the remaining images are ignored. In our case, we mainly use two colours i.e.: red and green. Therefore only these colours will be recognized and the remaining images which do not contain these colours will be ignored or removed. Nor the fingers of the user nor any other background colour will be shown in the output images of colour recognition process. For this purpose, the RGB colours are set prior in the MATLAB code, so that no other code except those which are set in the code will be detected. The output images are displayed in continuation and at the same speed as the speed at which slicing of video is done, so that it looks like a continuous movie in which the input is physical world and the output is only those colours which are present at the fingertips of the user. The colour is associated with the mouse cursor on the screen in such a way that as the colour caps move in the output images, the mouse cursor on the screen also moves in the same manner with respect to the users fingers.

\section{OBSERVATION}

Initially we use two colours which act nothing but act as the process of the mouse. Using MATLAB, we assign specific code and set each colour with that specific task. For e.g.: red colour is set for the cursor movement on the screen, Green colour for double click. Coloured caps or markers are attached at the finger tips of the user to operate the computer cursor. Marking the user's fingers with different colour caps helps the webcam to recognize the gestures made by the fingers. The movements and arrangements of these markers are grasped as gestures that act as an instruction for the projected application interfaces.

Web Camera acts as a digital eye connecting the physical world to the digital world. Web Camera is used to capture the image of object in sight range and follow the user's hand gestures. The Camera takes the live video as input in a 
continuous manner and then sends it to the laptop containing MATLAB installed in it.

Now, the MATLAB installed in the laptop processes the input images and recognizes the colour present on the finger tips of the user using gesture recognition. In MATLAB, code is prepared which convert the incoming live video from camera into frames of images or slicing of video is done in the form of images. The images obtained from this process are then processed further for colour recognition. Only the RGB colours that are set prior in MATLAB code will be detected in the colour recognition process.

The output images are displayed in continuation and at the same speed as the speed at which slicing of video is done, so that it looks like a continuous movie in which the input is physical movement of the fingers and the output is only those colours which are present at the fingertips of the user. The colour is matched with the cursor movements in the code, and alternately the cursor moves on the screen, producing the output. Thus, the mouse clicks are replaced with our finger clicks using object detection, colour recognition and image processing.

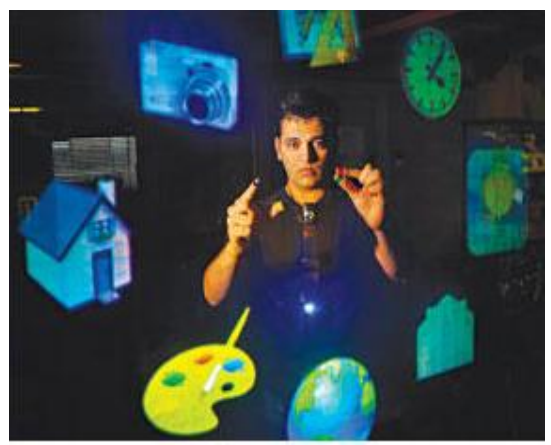

Figure: 7 Control PC with mouse cursor

\section{CONCLUSION}

The system architecture that we have proposed will completely change the way people would use the Computer system. Presently, the webcam, microphone and mouse are an integral part of the Computer system. Our product which uses only webcam would completely eliminate the mouse. Also this would lead to a new era of Human Computer Interaction (HCI) where no physical Contact with the device is required. The use of object detection and image processing in MATLAB for the implementation of our proposed work proved to be practically successful and the movement of mouse cursor is achieved with a good precision \& accuracy [12].

\section{FUTURE WORKS}

This paper is basically based on the development of the mouse cursor movement using fingers \& various colour markers on the tips. The efficiency \& usability of the system is very high, especially for its use with Laptop \& Desktop applications. It exhibits accuracy and speed, which are sufficient for many real time applications. However, in future we can add several other applications with it such as enlarging and shrinking windows, creating new icons/folders on the desktop, closing window, Direct image capturing, email browsing etc. by using multiple fingers with different gestures[10].

\section{ACKNOWLEDGEMENTS}

We would like to express our sincere heartfelt gratitude to Dr. Sraban Mukherjee (Director), and Mr. Narbada Prasad Gupta (Associate Professor \& Head of Department of Electronics\& Communication Engineering), Ideal Institute of Technology, Ghaziabad, for their helpful \& encouraging attitude all the time. We are very much grateful to our honourable esteemed guide Mr. Ajit Kr Singh, Assistant Professor, Department of Electronics and Communication Engineering for his kind and valuable guidance in the successful completion of this project work.

\section{REFERENCES}

[1] International Journal of Engineering Science \& Advanced Technology Volume-2, Issue-2, 245 - 249

[2] Chin, C. A., Barreto A., "Enhanced Hybrid Electromyogram/Eye Gaze tracking Cursor Control system Hand Free Computer Interaction", IEEE Conference Publication, 2006, pp: 2296-2299

[3] "Decoding Hand Cursor Kinematics Magnet Encephalographic Signals During tool Use"/Bradberry T. J., Contreas-Vidal, J.L., Rong Feng/2008,Page(s):53065309 .

[4] Dong Ming, Yuhuan Jhu, Hongzhi Qi, Baikun Ba, "Study On EEG-Based Mouse system By Using Brain Computer Interface", VECIMS-2009, International conference on virtual Environments, Human Computer interfaces and Measurements systems, Honkong China, May 11-13,2009

[5] Virtual mouse vision based interface, Robertson P., Laddaga R., Van Kleek M.

[6] HAN, M. H. , JANG, D. The Use of maximum curvature points for the recognition of partially occuladed objects. Pattern Recognition 1990, vol. 23 No.1/2, p. 21-23.

[7] MANJUNATH, B. S. , SALEMBIER, P., SIKORA, T. Introduction to MPEG-7 Multimedia Content Description Interface. March 2003, ISBN:0-471-486787.

[8] Qiu,"Color Image Indexing Using BTC" , IEEE Transactions on Image Processing, Volume 12, Number 1, pp. 93-101, January 2003.

[9] Craig A. Chin, and Armando Barreto, "Enhanced Hybrid Electromyogarm/Eye Gaze Tracking Cursor Control System for Hands Free Computer Interaction", Proceedings of $28^{\text {th }}$ IEEE EMBS Annual International Conference, New York City, USA,August 30-sep 3, 2006.

[10] International Journal of Engineering Research \& Technology (IJERT), ISSN 2278-0181,vol. 2 , Issue 2, February 2013. 